\title{
THE LOS ALAMOS NATIONAL LABORATORY PLASMA WAKEFIELD EXPERIMENT*
}

\author{
S. J. Russell ${ }^{\dagger}$, J. D. Goettee and B. E. Carlsten, LANL, Los Alamos, NM 87545, USA
}

\begin{abstract}
The electron beam from the Sub-Picosecond Accelerator at Los Alamos National Laboratory has been used as the drive beam in an electron beam driven, plasma wake-field accelerator experiment. The electron beam was directed into a plume of xenon gas from a supersonic jet. After the first two micro-pulses in the electron beam ionized the xenon, subsequent beam bunches experienced strong wake-field effects. The charge per bunch for our electron beam varied from $1-2 \mathrm{nC}$ and the pulse length of our micro-pulses was approximately 2 ps root-meansquared (rms). We observed deceleration gradients from the plasma wakefields as high as $74 \mathrm{MV} / \mathrm{m}$ over a $3 \mathrm{~mm}$ interaction length. In this paper we present our current results and future goals of the experiment.
\end{abstract}

\section{INTRODUCTION}

At the Los Alamos National Laboratory SubPicosecond Accelerator facility (SPA), the electron beam from an $8 \mathrm{MeV}$, radio-frequency photoinjector operating at $1300 \mathrm{MHz}[1]$ is longitudinally compressed in a magnetic chicane[2]. Most recently the SPA beam has been used as the drive beam in an electron beam driven plasma wake-field accelerator experiment (PWFA).

\section{EXPERIMENTAL SETUP}

\subsection{Electron Beam}

Table 1 gives the general properties of the SPA accelerator and electron beam. For the PWFA experiment, this beam is directed into a supersonic gas jet, as diagramed in Figure 1. The electron beam format is that of a macro-pulse containing one to ten micro-pulses. The repetition rate of the macro-pulse is typically $1 \mathrm{~Hz}$. The micro-pulses are spaced 9.2 ns apart.

Table 1: SPA photoinjector properties.

\begin{tabular}{|l|l|}
\hline Operation frequency & $1.3 \mathrm{GHz}$ \\
\hline Number of cells & $51 / 2$ \\
\hline Beam energy & $7-8 \mathrm{MeV}$ \\
\hline Beam charge & $\leq 10 \mathrm{nC}$ per beam bunch \\
\hline Best normalized emittance & $2.5 \mathrm{~mm}$ mrad at $1 \mathrm{nC}$ \\
$(10$ ps beam bunch) & $5.0 \mathrm{~mm}$ mrad at $5 \mathrm{nC}$ \\
\hline \multirow{3}{*}{ Bunch length } & $\begin{array}{l}20 \mathrm{ps} \text { to less than } 1 \mathrm{ps} \\
\text { with magnetic } \\
\text { compression }\end{array}$ \\
\hline
\end{tabular}

*Work performed under the auspices of the U. S. Department of Energy.

†srussell@lanl.gov

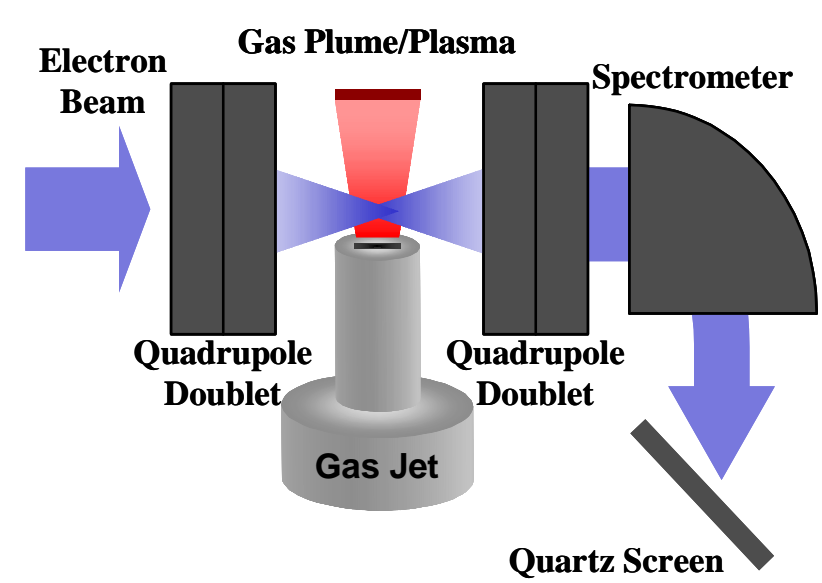

Figure 1: Diagram of PWFA experimental region. The plasma is formed in the gas plume.

\subsection{Supersonic Gas Jet}

Figure 2 is a picture of the supersonic gas jet. Its location in the experiment is shown in Figure 1. It was designed and built by Northrop-Grumman Corporation and has three rectangular nozzles, all $0.7 \mathrm{~mm}$ wide and of lengths 3,5 and $9 \mathrm{~mm}$. The length of the nozzles is parallel to the electron beam direction. Figure 3 shows the gas plume from the $9 \mathrm{~mm}$ long nozzle. Table 2 gives the relevant parameters for the gas jet plume.

Table 2: Properties of gas jet plume.

\begin{tabular}{|l|l|}
\hline Max. design inlet pressure & 760 Torr \\
\hline $\begin{array}{l}\text { Max. pressure differential between gas } \\
\text { jet inlet and gas jet outlet }\end{array}$ & $10^{5}$ \\
\hline $\begin{array}{l}\text { Gas density 1 mm downstream of orifice } \\
\text { at 760 Torr inlet pressure }\end{array}$ & $\begin{array}{l}\sim 5 \times 10^{19} \\
\text { atoms/cc }\end{array}$ \\
\hline $\begin{array}{l}\text { Gas density variation across } 400 \mathrm{micron} \\
\text { beam diameter in jet direction } 1 \mathrm{~mm} \\
\text { downstream from orifice }\end{array}$ & $<2$ \\
\hline $\begin{array}{l}\text { Gas density variation across jet orifice in } \\
\text { beam direction }\end{array}$ & $<2$ \\
\hline
\end{tabular}

\subsection{PWFA Experiment}

The compressed SPA electron beam is directed into the gas jet plume (Figure 1). After interaction with the gas jet, the beam is transported to a magnetic spectrometer where its energy spectrum is imaged.

The plasma in our PWFA experiment is formed in the gas plume. The supersonic gas jet is triggered for 10 milliseconds just before the electron beam is fired. The electron beam then ionizes the gas. We observe that after two micro-pulses have passed through the gas plume, 
plasma densities sufficient to cause significant energy loss in subsequent micro-pulses are obtained.

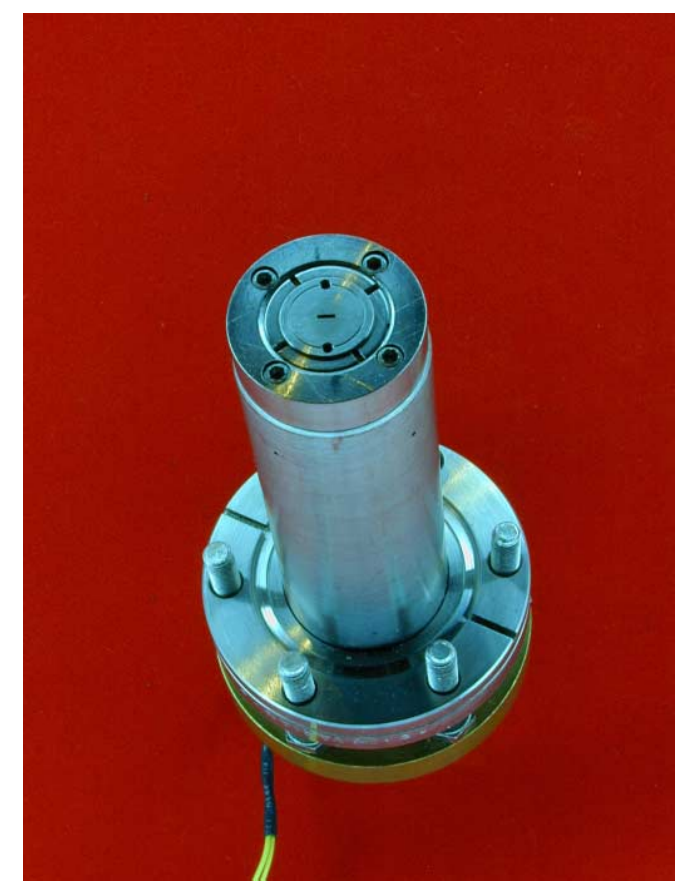

Figure 2: Picture of supersonic gas jet. Horizontal dimension (in picture) of the jet nozzle opening is $3 \mathrm{~mm}$.

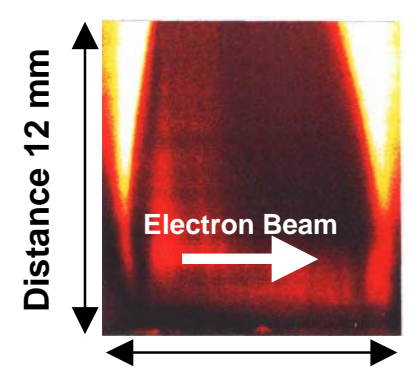

Distance $10 \mathrm{~mm}$

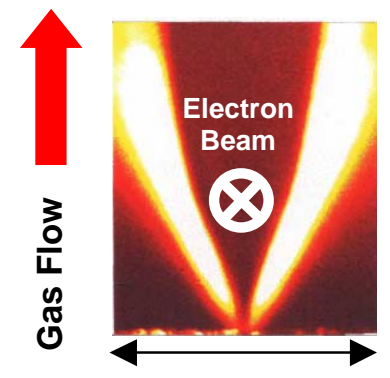

Distance $10 \mathrm{~mm}$
Figure 3: Gas plume for supersonic gas jet using $0.7 \mathrm{~mm}$ wide, $9 \mathrm{~mm}$ long nozzle. Bright regions show shock waves at edge of plume.

In our experiment, we used the $3 \mathrm{~mm}$ long gas jet nozzle and xenon gas. The inlet pressure on the gas jet was approximately 76 Torr, resulting in a neutral atom density of $\sim 5 \times 10^{17}$ atoms/cc in the interaction region. There was no plasma density diagnostic. The electron beam energy was $7.4 \mathrm{MeV}$ with 1 to $2 \mathrm{nC}$ of charge per bunch. From simulation, the bunch length of the beam pulses was $0.79 \mathrm{~mm}$ rms (2.6 pico-seconds temporally).
There was no bunch length diagnostic. The number of electron bunches per beam shot was varied from 1 to 5 .

\section{EXPERIMENTAL RESULTS}

In Figures 4 and 5 we show typical results from the experiment. Figure 4 shows spectrometer images (in false color) when the gas jet is off and when the gas jet is on for a five-bunch beam. The charge was $2 \mathrm{nC}$ per bunch in this case. As can be clearly seen, distinct energy loss was seen when the gas jet was operating. Profiles of the energy axis projection are overlaid on the images.

In Figure 5 we show energy profiles of the gas jet on case as we increased the number of bunches in the beam from one to five. Little or no energy loss is seen in bunches one and two. However, after the first two bunches, the gas jet is sufficiently ionized to cause dramatic energy loss in bunches three, four and five. Assuming the plasma length is the same as the gas jet nozzle length, $3 \mathrm{~mm}$, we can convert these energy losses to deceleration gradients. This is plotted in Figure 6, showing a peak deceleration gradient of $74 \mathrm{MV} / \mathrm{m}$ for bunch number four.
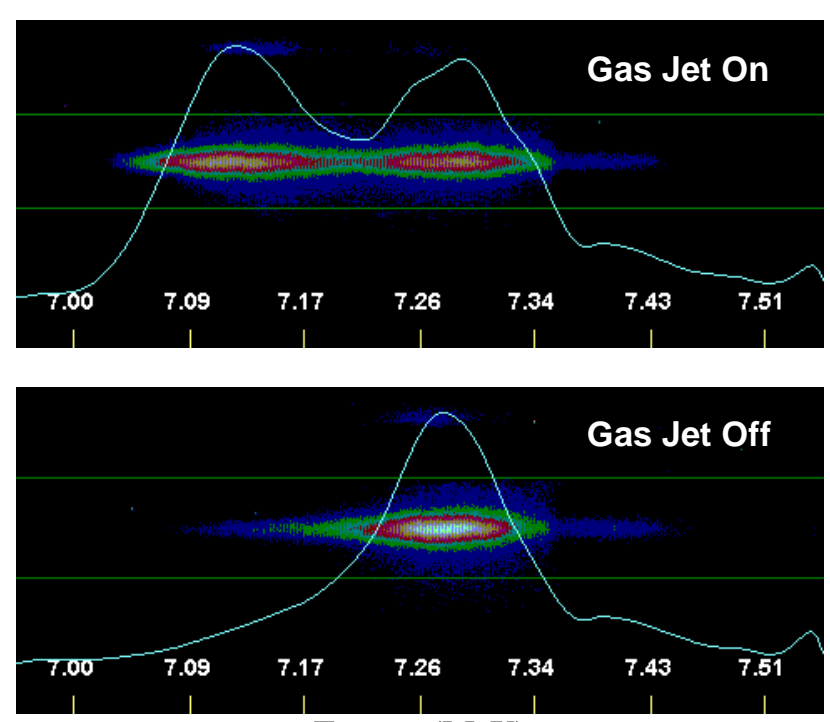

Energy (MeV)

Figure 4: Images of energy spectrometer for five-bunch SPA beam. The top image shows case when the gas jet is turned on. The bottom image shows the case when the gas jet is turned off. The first two beam bunches experience no energy loss. Bunches three, four and five see large plasma wake-fields and experience large energy loss. 


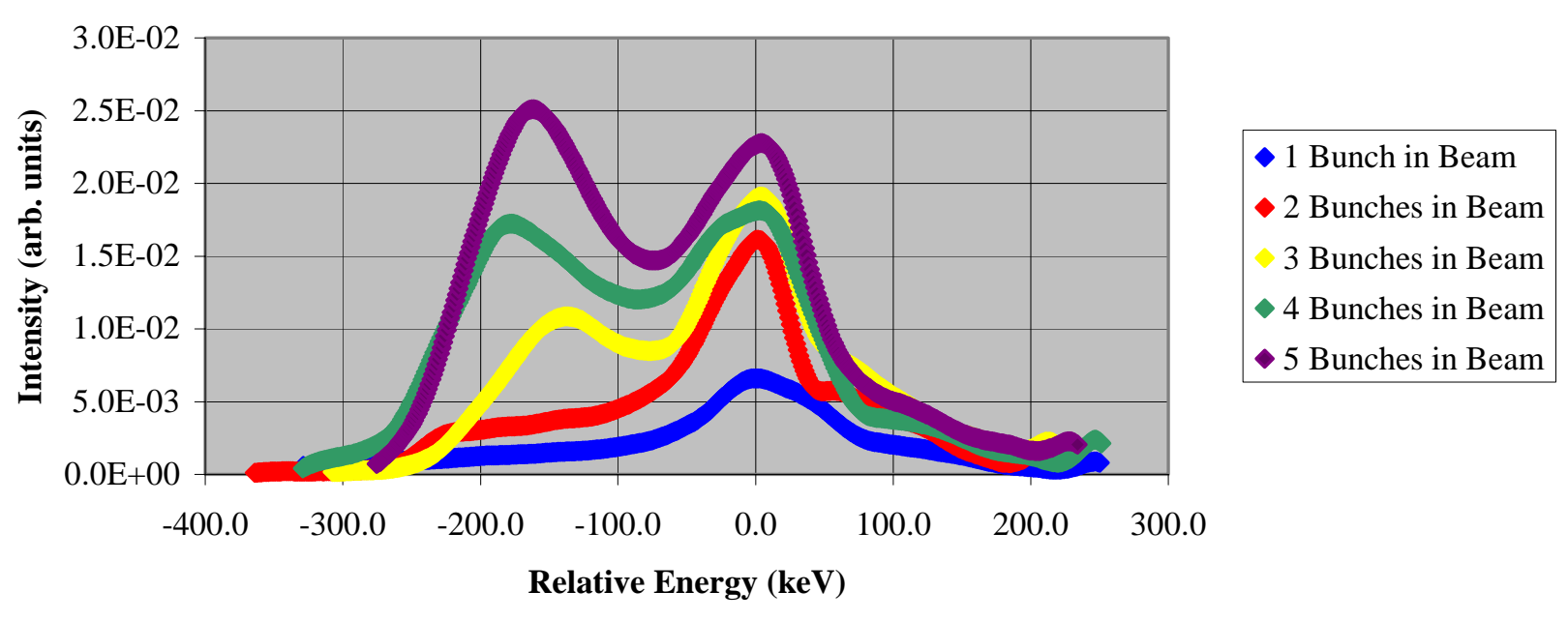

Figure 5: Measured energy spectrum of five electron beams after interaction with the gas jet plasma. The number of bunches in each beam was varied from one to five. The data clearly demonstrates the energy loss for bunches three, four and five.

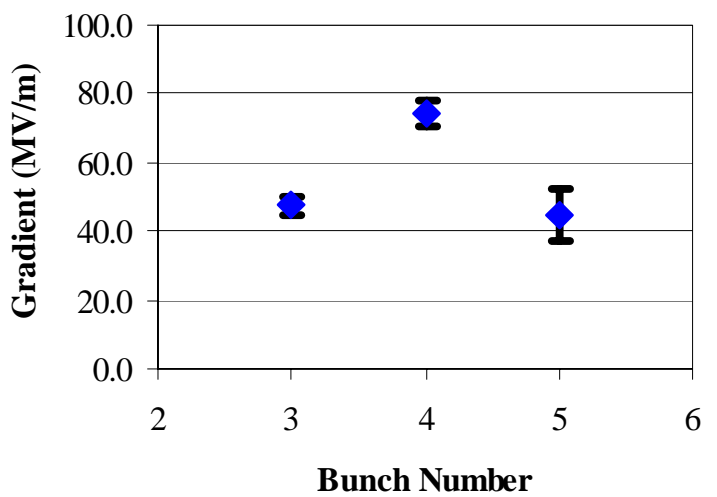

Figure 6: Measured plasma wake-field gradient versus bunch number for bunches 3, 4 and 5. Gas jet nozzle was $3 \mathrm{~mm}$ long. Bunch number 4 experienced the highest deceleration gradient, $74 \mathrm{MV} / \mathrm{m}$. Bunches three and five experienced lower deceleration gradients, presumable because the plasma density was too low and too high, respectively, for optimal plasma/beam coupling.

\section{CONCLUSION}

We have performed a PWFA experiment using only an electron drive beam. We have seen significant energy loss, indicating the presence of strong plasma wake-fields.

In the future, we hope to add a witness beam to measure the acceleration gradient of the plasma wake-fields. We also hope to add several diagnostic capabilities to the experiment, including an optical interferometer to measure our plasma density.

With proper tuning of our magnetic bunching scheme, we can create beam bunches with temporal lengths approaching $0.2 \mathrm{ps}$ rms[2]. This could result in at least a tenfold increase in the plasma wake-field magnitude[3].

\section{REFERENCES}

[1] B. E. Carlsten et. al., "Subpicosecond Compression Experiments at Los Alamos National Laboratory," Proceedings of the Micro Bunches Workshop, AIP Conf. Proc. 367, 21 (AIP, Upton, NY, 1995).

[2] B. E. Carlsten and S. J. Russell, "Subpicosecond compression of 0.1-1 nC electron bunches with a magnetic chicane at $8 \mathrm{MeV}$," Phys. Rev. E 53, 2072 (1996).

[3] J. B. Rosenzweig, "Particle Wake-field Accelerators," Proceedings of the 1992 Linear Accelerator Conference, 835 (AECL-10728, Chalk River, 1993). 